\title{
Intraoperative Fast 3D Shape Recovery of Abdominal Organs in Laparoscopy
}

\author{
Mitsuhiro Hayashibe ${ }^{1}$, Naoki Suzuki ${ }^{1}$, \\ Asaki Hattori ${ }^{1}$, and Yoshihiko Nakamura ${ }^{2}$ \\ 1 Institute for High Dimensional Medical Imaging, Jikei Univ. School of Med \\ 4-11-1, Izumihoncho, Komae-shi, Tokyo 201-8601 Japan \\ 2 Department of Mechano-Informatics, University of Tokyo \\ 7-3-1, Hongo, Bunkyoku, Tokyo 113-8656 Japan
}

\begin{abstract}
Precise measurements of geometry should accompany robotic equipments in operating rooms if their advantages are further pursued. For deforming organs including the liver, intraoperative geometric measurements play an essential role in computer surgery in addition to preoperative geometric information from CT/MRI. The laser-scan endoscope system acquires and visualizes the shape of the area of interest in a flash of time in laparoscopy. Results of in-vivo experiments on the liver of a pig verify the effectiveness of the proposed system. This system offers surgeons high-speed $3 \mathrm{D}$ geometric visualization to provide an intuitive orientation under laparoscopic surgery.
\end{abstract}

\section{Introduction}

Endoscopic surgery forces surgeons to operate under mental tension with mechanical and visual constraints. The visual difficulties are due to the narrow area of view of endoscopes and the lack of depth perception. This means surgeons are required to extract a sense of orientation in the abdominal cavity from the anatomical structure and the direction of the laparocope, which leads to awkward operating technique for surgeons. As a result this may cause you misperception due to the difference in environment from open surgery.

Laparoscopic surgery would be technologically improved if surgeons were provided with a $3 \mathrm{D}$ representation of the internal geometry in an intuitive manner. Being integrated with real time interface, such technology would make a significant difference in the operational environments. We believe this would be effective under surgery directly operated by surgeons and robotic surgery.

The technical advance of minimally invasive surgery has introduced more and more such equipped environments 1]. As there are many mechanical devices such as the laparoscope, with its holding arm and forceps, it would be essential to do a position management for these devices and organs from the viewpoint of safety. The 3D shape of the internal geometry can play an important role in enabling a surgeon to approach his target from the correct direction.

In this paper, we show the function of intraoperative fast $3 \mathrm{D}$ shape recovery system. The laser-scan endoscope acquires and visualizes the shape of the area of interest in a flash of time in laparoscopy. This system offers surgeons high-speed $3 \mathrm{D}$ geometric visualization to provide an intuitive orientation under laparoscopic surgery.

T. Dohi and R. Kikinis (Eds.): MICCAI 2002, LNCS 2489, pp. 356-363 2002.

(C) Springer-Verlag Berlin Heidelberg 2002 


\section{Intraoperative 3D Geometric Visualization}

Here, we depict the measured models by this system (first prototype). Please refer to the previous paper [6] as to system configuration. Figure 1 shows the scanned surface of a 50yen coin done by Laser-Pointing Endoscope. The hole on the 50yen coin could be measured and the error is within $1 \%$. The scanned 3D data is automatically redescribed in VRML 2.0 by our developed program. The surface is composed of numerous triangle patches.

The prime purpose of this research is to obtain 3D geometry under the laparoscopic surgery. We made an in-vivo experiment under the laparoscopy to obtain the intraoperative 3D geometry as shown in Fig.3. We scanned the area of $8 \mathrm{~cm}$ square and obtained 400 points of data. Sampling time took $1.2 \mathrm{~ms}$ for each point including the calculation time for the 3D position, and the total measuring time was 0.5 seconds. The intraoperative $3 \mathrm{D}$ geometry of the liver surface could be as quickly obtained as Fig.2.

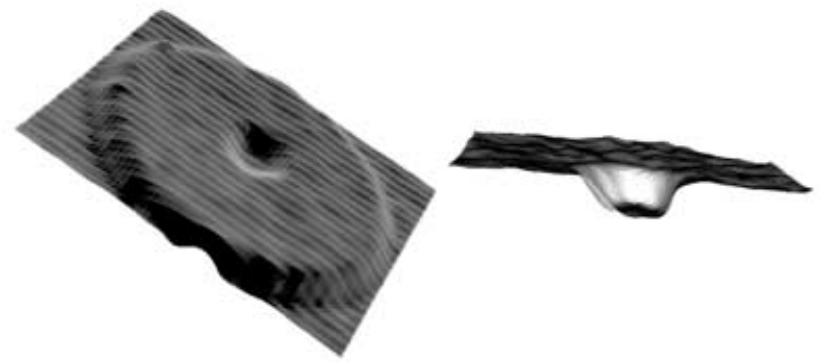

Fig. 1. Scanned surface of 50yen coin

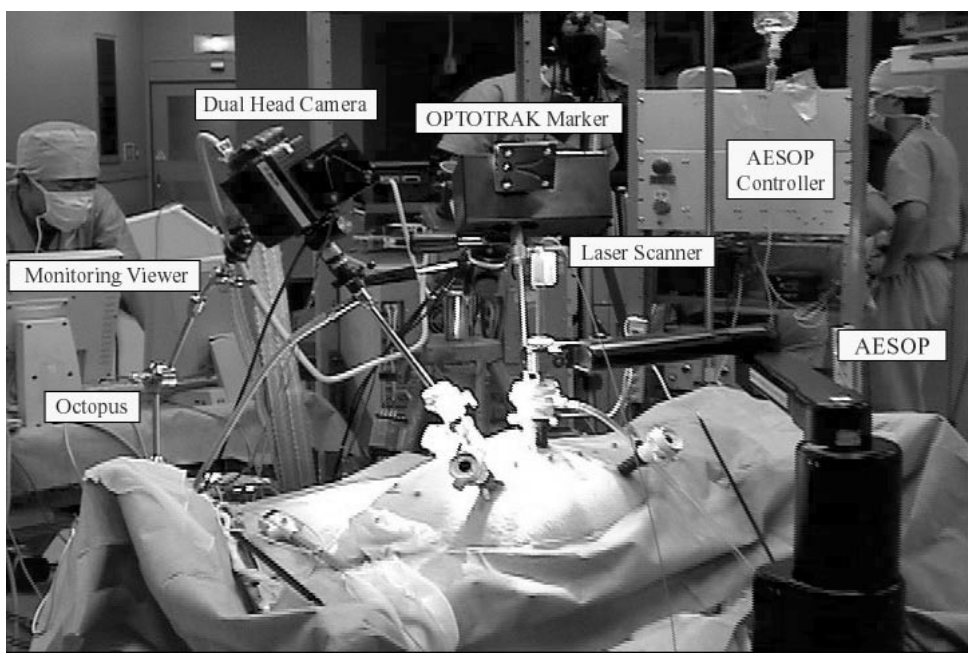

Fig. 2. The in-vivo experiment using pig's liver in laparoscopy 


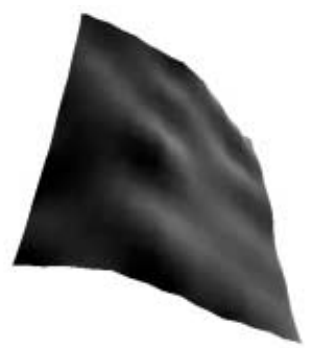

Fig. 3. Scanned VRML liver model in laparoscopy

\section{Extension for Real-Time Deformation Imaging}

The precise anatomical data of a patient is accessible using CT/MRI. The data is usually evaluated in the pre-operative conferences to discuss and plan the surgical procedures. One current issue is how to utilize such data in the operating room. The CT or MRI data shows geometrical inconsistency with the deformation due to the change of the patient's body postures. Regarding deformation, a surgeon has to rely on his imagination to map the pre-surgical data onto the deformed one. If we scanned the organ in-situ using the laser-scan endoscope, we would compute and estimate its internal deformation using the scanned data.

The first system we developed mainly focused on the surface geometric management. As a useful application, the effectiveness is shown in the field of robotic safety support[7]. However, visualization of the organ surface was not on-line. Now, we have developed an fast on-line $3 \mathrm{D}$ visualization system to show real-time deformation of organs under laparoscopy.

In laparoscopy, it is very difficult to get spatial perception. To supply a convenient interpretation in laparoscopy, we think it is useful to superimpose the deformed preoperative 3D model, which is reconstructed from $\mathrm{CT} / \mathrm{MRI}$, onto the actual organ in the laparoscopic image 8]. In order to deform the internal structure model of organ, the intraoperative $3 \mathrm{D}$ geometric information of the deformed organ should be required in real-time.

Fig.6 shows the system configuration of second prototype. Being controlled by the mirror of a galvano scanner, a laser line beam is projected inside the patient's body through an endoscopic optic device. We used a closed-loop galvano scanner which responds up to $1 \mathrm{kHz}$. The laser line pattern is captured by a $262 \mathrm{fps}$ highspeed camera (528*512 pixels, 256 gray scale) and a high-speed image processing board. The laser and camera coordinate systems are identified by OPTOTRAK attached to the devices. 3D coordinates of the reference lines are reconstructed based on the triangulation between the high-speed camera image and the mirror angles of the galvano scanner.

The image captured through the endoscope is distorted by the relayed lens inside. It is corrected and used to calculate the 3D position. The computed graphics are automatically produced from reconstruction of the scanned intraoperative 3D geometry. The feature of this system is on-line visualization of organ shape. We executed this task by parallel processing with 2 PCs. The task can 
be divided into measurement and visualization. Scanned points data is stacked into the shared memory board. The other PC gets out these points and makes polygons for the surface data. The scene is updated in OpenGL to visualize the deformation of object. Present frame rate of entire surface visualization is 5-6 frames per second(15 lines sampled in every frame), although we estimate that the processing time can be further shortened.

The concept of this system (second prototype) is to provide the surgeon with a completely intuitive interface. Therefore, the surgical navigation program is also being developed as Fig.6. The surgeon is provided with a laparoscopic view or another favored point of view to see the intraoperative liver deformation.

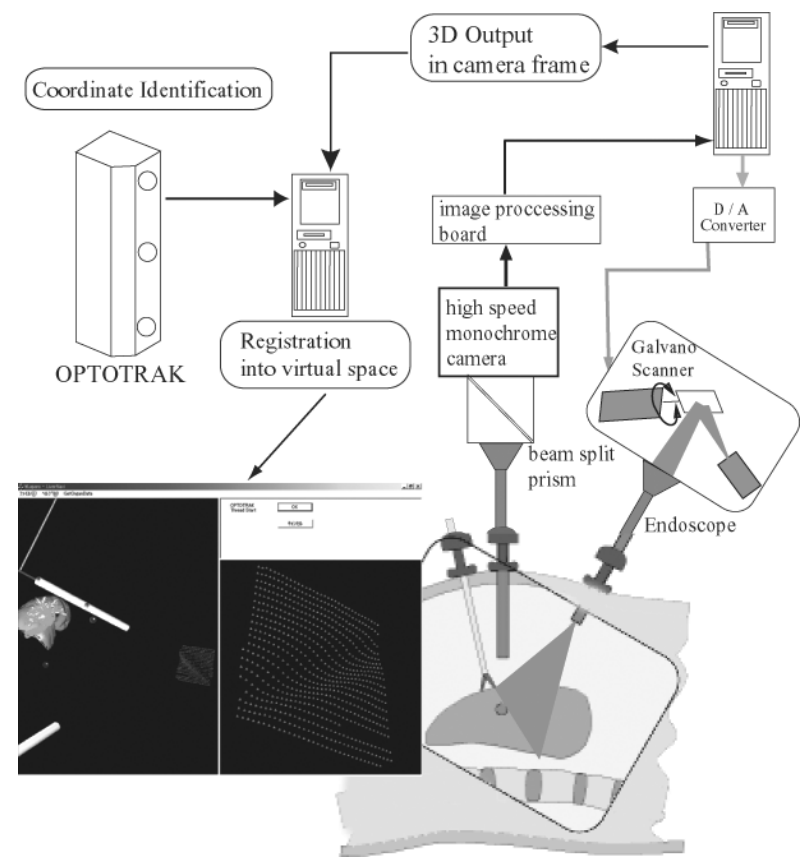

Fig. 4. System configuration

\section{Auto-polygon Generation from Range Data}

The laser-scan endoscope first outputs range data set of scanned organ. When an effective visualization of organ shape are performed using this range data, the surface should be composed from numerous triangle patches. These combination of points which form the triangles can be used for geometric computation to check the collision between an objective organ and equipped medical tools such as forceps which position is measured. We developed the program which automatically generates triangular patches from range data every frame.

In view of a laser scanner side, range data is a gathering of points mostly located in a lattice as shown in Fig 5 (left). When closely neiboring three points exist in this ordered set of points, the surface is constituted as a gathering of triangles of these three points. If the number of sampled points in a line is $n D o t$ 
and the number of scanned lines is nLine, The combination of vertexes of upper triangle is

$$
(i * n \operatorname{Dot}+j,(i+1) * n D o t+j, i * n D o t+j+1)
$$

as to lower triangle,

$$
((i+1) * n D o t+j,(i+1) * n D o t+j+1, i * n D o t+j+1)
$$

Related with column $i=0,1,2, \ldots . . n L i n e-1$; related with row $j=0,1,2, \ldots$ nDot- 1 , The combination of vertexes of triangle patch is decided repeatedly. Fig 5 (right) depicts the surface model transformed by this algorithmDThe condition of unevenness is easily perceived from shading and the sequentially scanned images.

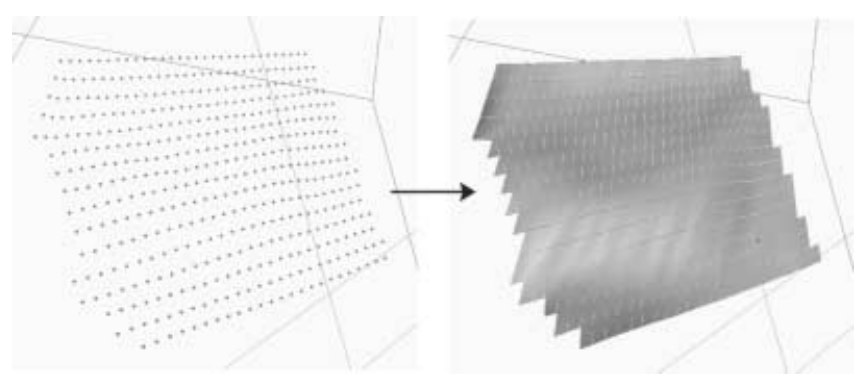

Fig. 5. Auto Polygon Generation

\section{Results}

\subsection{Deformation Imaging}

Figure 6 shows the result of deformation imaging at the laboratory. We scanned the deformation of the pinned cloth pulled by a string. Reconstructed polyhedron in the virtual space deformed in real-time in accordance with how the cloth was pulled by the string. The scanning area was $9 \mathrm{~cm}$ by $9 \mathrm{~cm}$.
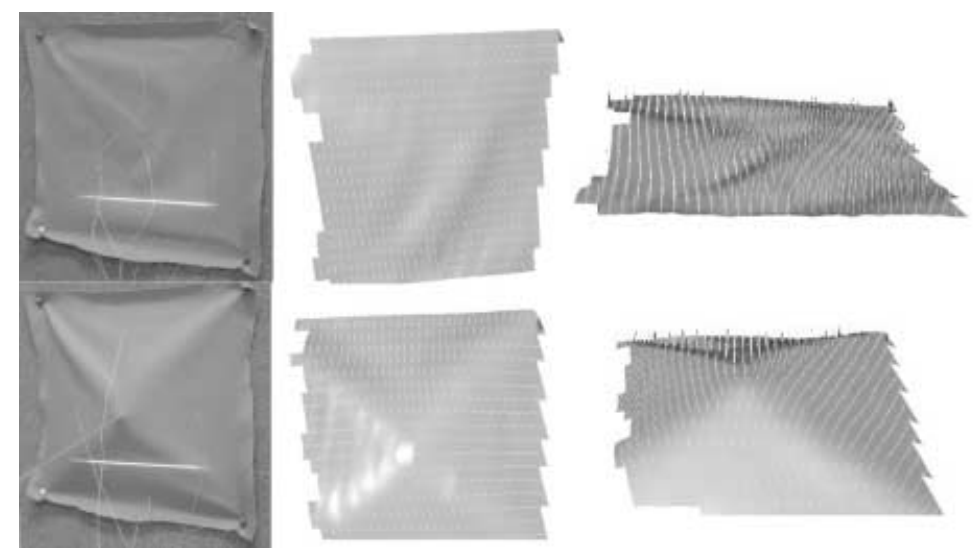

Fig. 6. Real-time Deformation Imaging left:video image middle:scanned surface data(view1) right:scanned surface data(view2) 
The frame rate of surface visualization actually depends on the scanning resolution. In rough scan mode, the number of sampled lines are decreased to accelarate the refresh cycle. If a fine surface measurement is required, you can change scanning settings from GUI of our developed prgram. In rough scan mode, we usually set the sampling number of scan line 15 . In this case, its frame rate is 6-7fps. In other words, 100 lines per second are able to be captured in this system. Even in rough mode, it is enough to perceive the entire shape of object.

Next, we tested the measurement and the visualization of the surface of an isolated pig's liver as shown in Fig.7 The laser scope and the camera scope are positioned at the distance of $15 \mathrm{~cm}$ from the organ. Even in the shinny and wet surface condition, the image processing of high-speed camera was succeed and the deformation of organ surface could be observed with the rendered computer graphics.

An incision was made on the liver surface in Fig 8 . The length of the incision is $44 \mathrm{~mm}$ and the depth $7 \mathrm{~mm}$. Figure 9 depicts the image of reconstructed surface of liver in one frame. The scanning area is $8 \mathrm{~cm}$ by $6.5 \mathrm{~cm}$. The yellow lines are normal vectors. In Fig,10, one more incision was made in the crossing position of the former. The incision with the length of $42 \mathrm{~mm}$ and the depth of $5 \mathrm{~mm}$ was addedon the surface. Even on this uneven surface, the visualization of liver deformation has been successfully done in Fig[11.

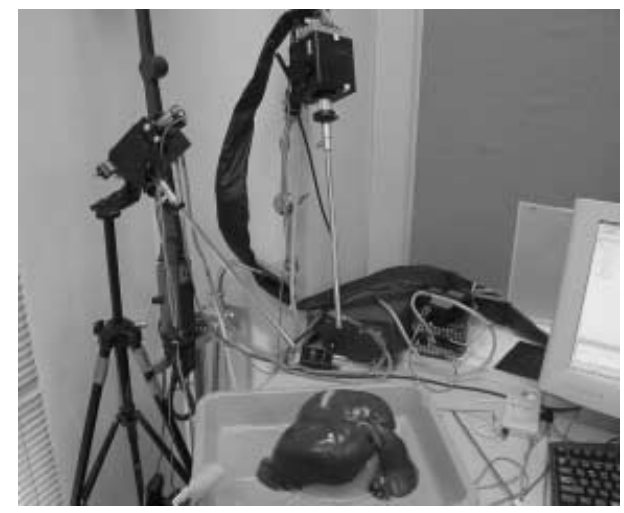

Fig. 7. Real-time Deformation Imaging Experiment in in-vitro
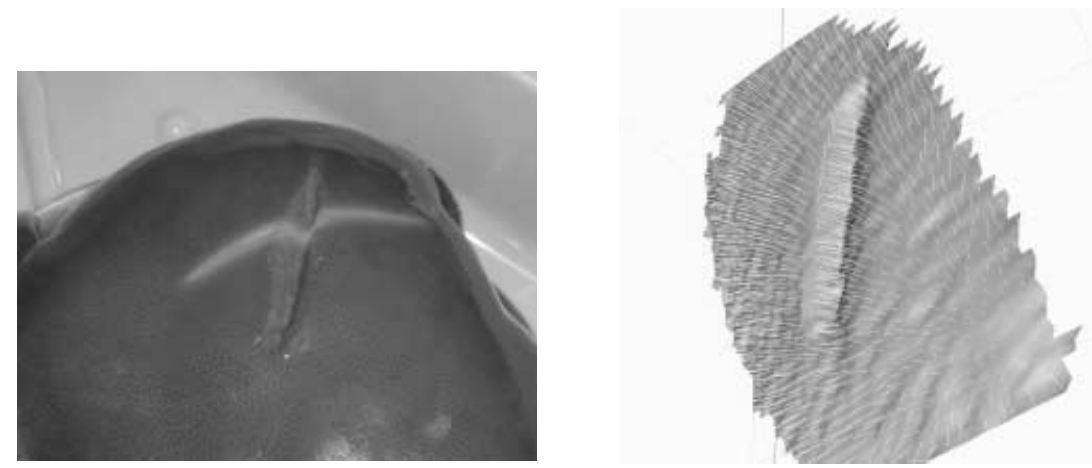

Fig. 8. Incision on Liver Surface(one in- Fig. 9. Visualization of the Shape of Incision) cised Organ(one incision) 


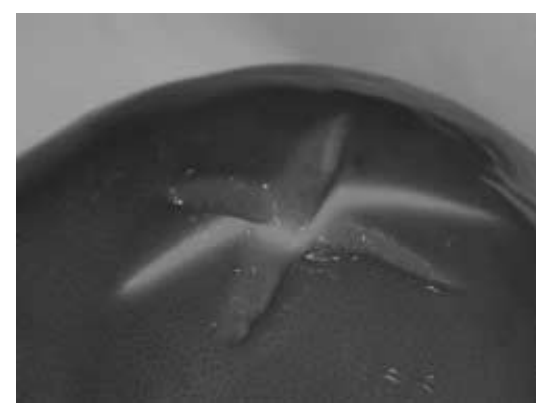

Fig. 10. Incision on Liver Surface(crossed Fig. 11. Visualization of the Shape of Inincision)

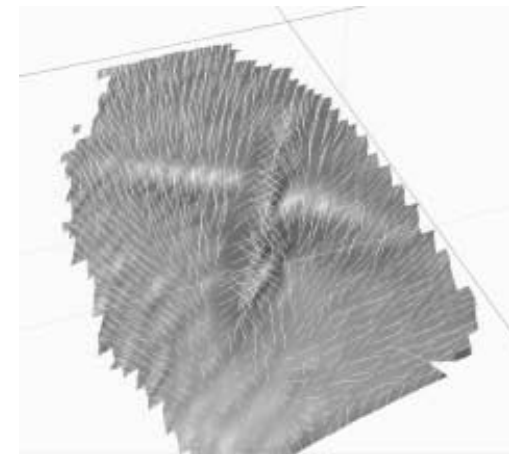

cised Organ(crossed incision)

\subsection{Registration of Preoperative 3D Organ Model}

The precise anatomical data of a patient is accesible using CT or MRI. The data is usually evaluated in the pre-operative conferences to discuss and plan surgical procedures. One of current issues is how to utilize such data in the opetarion room. The CT or MRI data shows geometrical inconsistency with the deformation due to the change of patient's body postures. For deformation, a surgeon has to develop the imagination to map the pre-surgical data onto the deformed one.

As first step, we tested the registration of preoperative 3D model assuming thatthe organ is rigid. Here, preserved human liver in formalin was used. Figure 12 shows the plaster cast of the liver scanned by this system. As preoperative model, CT slices of preserved liver was reconstructed in 3D model. To correspond the 3D model with in-situ scanned surface of plaster cast, a few points are manually chosen as feature points from scanned surface. Here, anatomical feature points around gallbladder are used. Figure 13 shows the fitted 3D liver model onto scanned surface. This system can be applied for the navigation in laparoscopy because the position of scope and forceps is also observed and displayed continuously. For the future, we plan to take into account of the elasticity of organ.

\section{Conclusion}

The conclusion of this paper is summarized in the following two points:

- The intraoperative geometric information of the internal surface in laparoscopy was obtained successfully in the pig experiment. Preliminary results of in-vivo experiments verified the functionality and showed the performance.

- Real-time deformation imaging has been realized using parallel processing and auto polygon generation. Reconstructed polyhedron in the virtual space deformed in real-time in accordance with how the shape of the object changed in the experiment using an isolated pig's liver. 


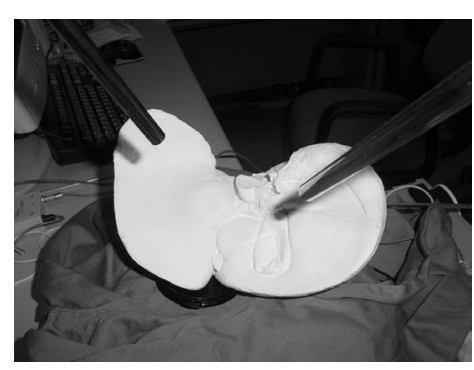

Fig. 12. Plaster Cast of Human Liver

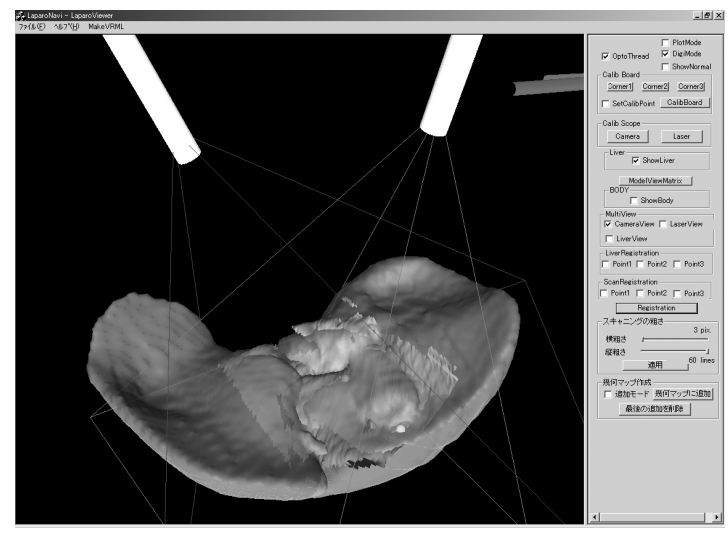

Fig. 13. Registered 3D Liver Model onto Scanned Surface

\section{References}

1. Marc O. Schurr.: Robotic devices for advanced endoscopic surgical procedures. Journal of the Robotics Society of Japan. Vol. 18 No. 1 (2000) 16-19

2. Guthart GS, Salisbury JK.: The Intuitive Telesurgery System. Proceedings of the IEEE International Conference on Robotics and Automation. (2000) 618-621

3. Computer Motion Inc..: Internet Home Page. http://www.computermotion.com.

4. Y.Nakamura, M.Hayashibe.: Laser-Pointing Endoscope System for Natural 3D Interface between Robotic Equipments and Surgeons. Proceedings of Medicine Meets Virtual Reality 2001. (2001) 348-354

5. Eric Larsen, Stefan Gottschalk, Ming Lin and D.Manocha.: Fast proximity queries with swept sphere volumes. Technical report TR99-018, Department of Computer Science, UNC Chapel Hill, (1999)

6. M. Hayashibe, Y. Nakamura.: Laser-Pointing Endoscope System for Intraoperative 3D Geometric Registration. 2001 IEEE International Conference on Robotics and Automation. (2001) 1543-1548

7. M. Hayashibe, Y. Nakamura, H. Shimizu and M. Okada. A Laser-Pointing Endoscope System Providing the Operational Support of Surgical Robot. Proc. of the 32nd International Symposium on Robotics 2001, pp.636-641, 2001.

8. A. Hattori, N. Suzuki, M. Hashizume, T. Akahoshi, K. Konishi, S. Yamaguchi, M. Shimada, M. Hayashibe. Development of Data Fusion System for Robotics Surgery(da Vinci). Journal of Japan Society of Computer Aided Surgery., Vol.18 No.1Cpp.45-48, 2002D

9. Yulun Wang. The Evolving Role of Robotics In Surgery. Journal of the Robotics Society of Japan.CVol.18 No.1Cpp.45-48, 2000D

10. H.Haneishi, Y.Yagihashi, Y.Miyake. A new method for distortion correction of electronic endoscope images. IEEE Trans. Med. Imag., Vol. 14, pp. 548-555, September 1995.

11. H. Haneishi, T. Ogura, Y. Miyake. Profilometry of a gastrointestinal surface by an endoscope with laser beam projection. Optics Letter., Vol.19 No.9, pp. 601-603, 1994. 